The inverse problem of sensing the mass and force induced by an adsorbate on a beam nanomechanical resonator

Yun Liu and Yin Zhang

Citation: AIP Conference Proceedings 1738, 480016 (2016); doi: 10.1063/1.4952252

View online: http://dx.doi.org/10.1063/1.4952252

View Table of Contents: http://aip.scitation.org/toc/apc/1738/1

Published by the American Institute of Physics 


\title{
The Inverse Problem of Sensing the Mass and Force induced by an Adsorbate on a Beam Nanomechanical Resonator
}

\author{
Yun Liu and Yin Zhang ${ }^{\mathrm{b}}$ \\ ${ }^{a}$ Faculty of Information and Automation, Kunming University of Science and Technology, Kunming, Yunnan \\ Province 650051, China \\ ${ }^{b}$ State Key Laboratory of Nonlinear Mechanics, Institute of Mechanics, Chinese Academy of Sciences, Beijing, \\ 100190, China
}

\begin{abstract}
The mass sensing superiority of a micro/nanomechanical resonator sensor over conventional mass spectrometry has been, or at least, is being firmly established. Because the sensing mechanism of a mechanical resonator sensor is the shifts of resonant frequencies, how to link the shifts of resonant frequencies with the material properties of an analyte formulates an inverse problem. Besides the analyte/adsorbate mass, many other factors such as position and axial force can also cause the shifts of resonant frequencies. The in-situ measurement of the adsorbate position and axial force is extremely difficult if not impossible, especially when an adsorbate is as small as a molecule or an atom. Extra instruments are also required. In this study, an inverse problem of using three resonant frequencies to determine the mass, position and axial force is formulated and solved. The accuracy of the inverse problem solving method is demonstrated and how the method can be used in the real application of a nanomechanical resonator is also discussed. Solving the inverse problem is helpful to the development and application of mechanical resonator sensor on two things: reducing extra experimental equipments and achieving better mass sensing by considering more factors.
\end{abstract}

Keywords: Resonator; resonance frequency; Galerkin method.

PACS: $46.40 . \mathrm{Ff}, 62.40 .+\mathrm{I}$

\section{INTRODUCTION}

Mass spectrometry is a widely used analytical tool in biology and chemistry, which is also expected to play an important role in proteomics $[1,2]$. However, whether mass spectrometry can be the mainstay instrument in proteomics is questionable $[2,3,4]$. During the application of mass spectrometry, there are three stages: ionization, separation and detection [5]. The structural change of protein [3] or damage of fragile biological macromolecules [6] caused by ionization is a serious problem in the application of mass spectrometry. Mass spectrometry also has the problem of being applied to small and thermostable compounds because of the difficulty of ionization and transferring ionized analytes from the condensed phase into the gas phase [2]. On the other hand, the sensing mechanism of a mechanical mass resonator is the shifts of resonant frequencies, which can work with the electrically neutral analytes. The first two stages of ionization and separation are thus unnecessary for a mechanical mass resonator [4].

\section{MODEL DEVELOPMENT}

When an adsorbate is on a carbon nanotube (CNT)-based resonator with the length of $L$, the governing equation of the resonator which is modeled as a beam is given as follows $[7,8]$

$$
\left[m+M_{o} \delta\left(x-x_{o}\right)\right] \frac{\partial^{2} w}{\partial t^{2}}-T \frac{\partial^{2} w}{\partial x^{2}}+D \frac{\partial^{4} w}{\partial x^{4}}=0 .
$$

Where $m$ is the resonator mass per unit length; $M_{o}$ and $x_{o}$ are the mass and position of the adsorbate, which is modeled as a concentrated mass by the Dirac delta function of $\delta[7,8] . w$ is the beam displacement; $T$ is the axial load, $T>0$ is tension and $T<0$ is compression. $D$ is the beam bending stiffness and $D=E I$ ( $E$ and $I$ are the beam Young's modulus and the moment of inertia, respectively).

By introducing $\xi=x / L, \tau=\sqrt{E I /\left(m L^{4}\right)} t$ and $W=w / L$ [7], Eq. (1) is nondimensionalized as follows 


$$
\left[1+\alpha \delta\left(\xi-\xi_{o}\right)\right] \frac{\partial^{2} W}{\partial \tau^{2}}-\beta \frac{\partial^{2} W}{\partial \xi^{2}}+\frac{\partial^{4} W}{\partial \xi^{4}}=0 .
$$

Where the dimensionless parameter $\alpha=M_{o} /(m L)$ is the ratio of the adsorbate mass to that of the resonator; $\beta=T L^{2} / D$ is the ratio of the axial load to the beam transverse stiffness; $\xi_{o}=x_{o} / L$ is the adsorbate location. When the compressive axial load reaches a critical value, the beam buckles. Eq. (2) is a linear equation, which cannot describe the beam vibration in the post-buckling region. For Eq. (2) to apply, $\beta>-4 \pi^{2}$ for the clampedclamped beam and $\beta>-\pi^{2} / 4$ for the cantilever beam are required.

The Galerkin method is an efficient method for the eigenfrequency computation of a beam with small concentrated masses 40 , which assumes the following form for $W(\xi, \tau)$

$$
W(\xi, \tau)=\sum_{j=1}^{N} a_{j}(\tau) \phi_{j}(\xi),
$$

where $N$ is the mode number and $a_{j}(\tau)$ is the unknown jth modal amplitude. $\phi_{j}(\xi)$ is the jth mode of a uniform clamped-clamped beam. Substitute Eq. (3) into Eq. (2), time $\phi_{i}(\xi)$ and integrate from 0 to 1 , the following governing equations are derived

$$
\mathbf{M} \ddot{\mathbf{q}}+\mathbf{K q}=0
$$

Here $\dot{()}=\partial / \partial \tau$ and $\mathbf{q}$ is a vector given as $\mathbf{q}=\left(a_{1}, a_{2}, \cdots \cdots, a_{N}\right)^{T} . \mathbf{M}$ and $\mathbf{K}$ are the $N \times N$ matrices of mass and stiffness, respectively, which are given as the following by using both the orthonormality property of $\phi_{j}(\xi)$ and the integration property of the Dirac function $[7,8]$ :

$$
\mathbf{M}_{i j}=\delta_{i j}+\alpha \phi_{i}\left(\xi_{o}\right) \phi_{j}\left(\xi_{o}\right), \quad \mathbf{K}_{i j}=\kappa_{j}^{4} \delta_{i j}-\beta \int_{0}^{1} \phi_{i}(\xi) \frac{\partial^{2} \phi_{j}(\xi)}{\partial \xi^{2}} d \xi .
$$

Where $\delta_{i j}$ is the Kronecker delta function and it is noticed that the presence of the concentrated mass $(\alpha)$ makes the mass matrix non-diagonal; $\kappa_{j}^{2}$ is the jth (dimensionless) eigenfrequency of a uniform undamped beam with no axial load. Clearly, the presence of the axial load $(\beta)$ has the direct impact on the stiffness matrix $\mathbf{K}$, which also leads to the variation of the resonant frequencies. The first three $\kappa_{j}^{2}$ of a clamped-clamped beam are given as follows:

$\omega_{1}^{o}=\kappa_{1}^{2}=4.73^{2}=22.3733, \quad \omega_{2}^{o}=\kappa_{2}^{2}=7.8532^{2}=61.6728, \quad \omega_{3}^{o}=\kappa_{3}^{2}=10.9956^{2}=120.9034$

To find out the resonant frequencies of the beam with the concentrated mass and axial load, $a_{j}(\tau)=b_{j} e^{i \omega \tau}$ ( $b_{j}$ is the unknown constant and $\omega$ is the resonant frequency) is assumed and substituted into Eq. (4), which leads to the following eigenvalue problem

$$
\mathbf{K}-\mathbf{M} \omega^{2}=0 .
$$

To find the eigenfrequency/resonant frequency of $\omega$, the adsorbate mass $(\alpha)$, location $\left(\xi_{o}\right)$ and axial load $(\beta)$ are needed. Here up to three resonant frequencies are calculated, $N \geq 3$ is required. By carefully choosing the lower and upper bounds for each resonant frequency, different $\omega$ is solved one by one by the Newton-Rhaphson method [8]. 


\section{RESULTS AND DISCUSSION}

Now let us present how to use the mechanism to solve the inverse problem. Here the computation example of $\alpha=$ $0.1, \xi_{o}=0.3$ and $\beta=10$ is given, which results in the following three resonant frequencies as given by Eq. (7)

$$
\omega_{1}=23.5217, \quad \omega_{2}=59.5752, \quad \omega_{3}=121.8384 .
$$

Tension stiffens the beam and thus increases the resonant frequencies; on the other hand, the adsorbate mass always reduces the resonant frequencies. Compared with the three resonant frequencies of $\alpha=\beta=0$ as given in Eq. (6), the competition between tension and mass leads to the decrease of the second resonant frequency and the increase of the first and third ones. In most case, the eigenfrequencies are solved as a forward problem by supplying $\alpha, \xi_{o}$ and $\beta$ into Eq. (7). However, in the real application of the resonator sensor, the resonant frequencies are the measured quantities which, in this computation example, are given in Eq. (8); $\alpha, \xi_{o}$ and $\beta$ in general are the unknowns to be determined. In order to present a better and graphic illustration on how the inverse problem is solved, we start with the simpler case of two variables. In this case, $\beta=10$ is known, $\alpha$ and $\xi_{o}$ are the two unknowns to be determined. Because the original axial load (or surface stress) can be determined during an experimental calibration process by measuring the shift of a resonant frequency [9], this inverse problem solving technique for two variables can correspondingly be applied to the case that adsorption induces no surface stress.

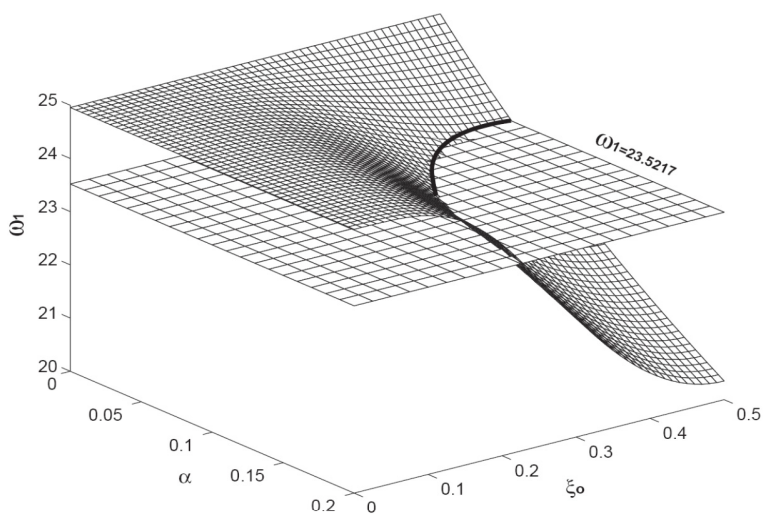

FIGURE 1. (a) The variation of the first resonant frequency $\left(\omega_{1}\right)$ as a function of $\alpha$ and $\xi_{o}$. The level plane is the one with the constant of $\omega_{1}=23.5217$. The intersection of the two planes is marked with a solid curve. Here the axial load is fixed as $\beta=$ 10 .

Figure 1 presents the variation of the first resonant frequency $\left(\omega_{1}\right)$ as the function of $\alpha$ and $\xi_{o}$. Here $\alpha$ varies from 0 to $0.2 ; \xi_{o}$ varies from 0 to 0.5 . Because the $\mathrm{C}-\mathrm{C}$ beam is a symmetric structure, the adsorbate at $\xi_{o}$ and 1 $\xi_{o}$ results in the same change for any arbitrary resonant frequency. Therefore, only half of the beam span is examined here. The level plane is the one with $\omega_{1}=23.5217$. The intersection of the two planes are marked with a solid line, which indicates the combinations of $\alpha$ and $\xi_{o}$ resulting the same first resonant frequency of $\omega_{1}=$ 23.5217. This solid line also indicates that the combinations are infinite. Figure 2 presents the variation of the second resonant frequency $\left(\omega_{2}\right)$ as the function of $\alpha$ and $\xi_{o}$. The level plane is the one with $\omega_{2}=59.5752$. Again, the intersection of the two planes is the combinations of $\alpha$ and $\xi_{o}$ resulting the same second resonant frequency of $\omega_{2}=59.5752$, which is marked a dashed line. Once again, the dashed line indicates that the infinite combinations of $\alpha$ and $\xi_{o}$ resulting the same second resonant frequency of $\omega_{2}=59.5752$. When $\alpha, \xi_{o}$ and $\beta$ are given, each eigenfrequency is uniquely determined by Eq. (7) as a forward problem. In comparison, in this two variables case of the inverse problem, for a given eigenfrequency, there are infinite combinations of $\alpha$ and $\xi_{o}$. However, when these 
two curves obtained in Figs. 1(a) and (b) are projected into the $\xi_{o} \quad \alpha$ plane, they intersect and the intersection point is marked as a circle, which is exactly $\left(\alpha, \xi_{o}\right)=(0.1,0.3)$. Physically, the reason for the two curves to intersect is that the mechanism mentioned above: $\alpha$ and $\xi_{o}$ have different impacts on different resonant frequencies; different resonant frequency responds differently to the given $\alpha$ and $\xi_{o}$. Mathematically, as seen in Eq. (5), $\alpha$ is a coefficient and $\xi_{o}$ is embedded in the function of mode shape in the mass matrix.

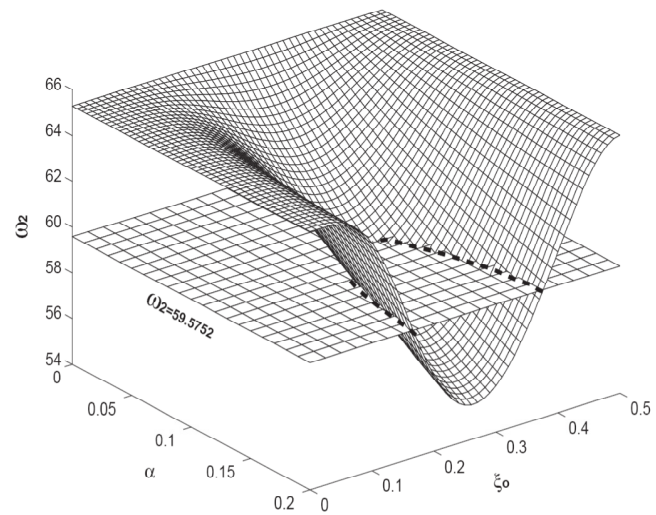

FIGURE 2. The variation of the second resonant frequency $\left(\omega_{2}\right)$ as a function of $\alpha$ and $\xi_{o}$. The level plane is the one with the constant of $\omega_{2}=59.5752$. The intersection of the two planes is marked with a dashed curve. Here the axial load is fixed as $\beta=10$.

\section{SUMMARY}

That the inverse problem can be solved is based on the following two facts: (1) Mass, position and axial load have different impact on a given resonant frequency; (2) for given mass, position and axial load, different resonant frequency varies differently. The graphic solution procedure for the two-variable case is presented and it can provide valuable information to guess the initial values for the Newton-Raphson method in the multiple-variable case.

\section{ACKNOWLEDGMENTS}

The research has been supported by the National Natural Science Foundation of China (NSFC No. 11372321).

\section{REFERENCES}

1. R. Aebersold, and M. Mann, Nature. 422, 198 (2003)

2. B. Domon, and R.Aebersold, Science. 312, 212 (2006).

3. M.S.Hanay, et al., Nat. Nanotech. 7, 602 (2012).

4. A.K. Naik,et al., Nat. Nanotech. 4, 445 (2009).

5. W. Hiebert, Nat. Nanotech. 7, 278 (2012).

6. R.G Knobel, Nat. Nanotech. 3, 525 (2008).

7. Y. Zhang, and K.D. Murphy, J. Sound Vibr. 330, 5569 (2011).

8. Y. Zhang, J. Vibr. Acoust. 133, 021006 (2011).

9. A.W. McFarland, et al., Appl. Phys. Lett. 85, 053505 (2005). 